\title{
Digital-Health Tourism Research-Methodology Coronavirus-Vaccination Trials: A Study Interpreting Geometa-Data Profiling to use Mobile-Health Technologies Nigeria
}

\author{
Wan Rozaini Sheik Osman', Hapini Awang², Abdullahi Hassan Birnin- \\ Kudu ${ }^{1,2,3 *}$
}

${ }^{1}$ Faculty of Computing and Information Technology,

Federal University Dutse, Dutse, Jigawa State, NIGERIA

${ }^{2}$ School of Computing,

Universiti Utara Malaysia, Kedah, 06010 Sintok, MALAYSIA

${ }^{3}$ Digital Technology Research Development Unit, Educational Division of Sarkin Kudu Hassan Jibril Memorial

Development Foundation, No.2 Bodan Street Birnin Kudu Jigawa State, NIGERIA

\section{*Corresponding Author}

DOI: https://doi.org/10.30880/emait.2021.02.02.005

Received 27 September 2021; Accepted 18 October 2021; Available online 15 November 2021

\begin{abstract}
Digital-Health Tourism Innovation (DTI) worldwide is in its infancy due to the emergent of coronavirus (COVID-19) disease. With the growth of open geometa data, use of government electronic services including electronic health (e-health), electronic commerce (e-commerce) and mobile health (m-health), Artificial Intelligence (AI) and machine learning strategies. Health and primary healthcare sectors are currently adopting these innovations for socio-economic wellbeing. Digital-health (also termed as e-health) is part of digital tourism innovation. Adapting geometa data profiling to develop a digital-health tourism framework for Primary Healthcare Workers (PHWs) to use mobile health technologies in COVID-19 vaccination trials are the key challenges of this study. Nevertheless, digital health tourism skills have been launched in developing Nations that created thousands of jobs to protect digital tourism businesses from potential vulnerabilities. Despite the benefits of this novel innovation, its deployment and implementation have been treated by inadequate of ICT facilities, lack of geometa data pre-processing to remove noise, data integrity, insufficient of academic research fundings, and reliable research methodology beyond COVID-19 vaccination trials to highlight these aspects. Therefore, qualitative, and quantitative research methods using Precaution Adoption Model Process (PAMP) questionnaire are employed to enable new ways of pre-processing behavior intention factors items. Eight academic researchers who were conversant with digital health technology validated 28 behavior intention factors with average factor loading values of $50 \%$ to $75 \%$. Pilot survey conducted among 700 respondents from March 18, 2020, to September 10, 2021, among them are undergraduate students that may use this technology for research purposes. Pre-processed geometa data have shown percentage frequency counts of internet access and other online services $8 \%$ to $95 \%$, adapted training factors $49 \%$ to $92 \%$ and factor items $34 \%$ to $78.3 \%$ for hypothesis generation towards development of digital health tourism framework in finding explanation to COVID-19 economic challenges. Except behavior intention factors and factor items insights are known and mapped, mobile health technology design process may result in poor conclusions. Thus, patients recovered from COVID-19 infection can still be infected again.
\end{abstract}

Keywords: Digital-health, tourism, healthcare-workers, geometa-data, coronavirus-vaccination, mobile-health, Nigeria 


\section{Introduction}

Adoption and adaption of behavior intention factors of self-alleged health, life fulfilment and connected factors among healthcare experts and widespread populace [1] can enable integration of theory and practice of health tourism and its economic impact [2] and can protect incoming and outgoing health tourists [3], improve health and healthcare big data usefulness [4] and biomedical ethics in health tourism [5] towards setting the standards for selecting analytical tools, forming different networks of patients, health and healthcare personnel that identifies diseases regardless of where these processes has taken place worldwide. This has inspired a pilot survey in defining behavior intention factors to support digital health tourism adoption success or failures in different contexts. Thus, this study labels various procedures to understand geometa data profiling that support geometa data profiles collection, guarantee strategic digital health tourism process without interruption, as in the case with the coronavirus (COVID-19) vaccination trials. Since, the traditional digital health tourism adoption models are based on analytical framework [2]. Nevertheless, this study adopts survey research design based on Precaution Adoption Model Process (PAMP) questionnaire, seven level sampling procedures are employed to pre-process health and healthcare information. While behavior intention models provides the theoretical framework. Similarly, the adoption of information and communication technologies (ICT), Artificial Intelligence (AI) and machine may be used to support digital health tourism framework to use mobile health technologies. Use AI and machine learning can improve health tourist activities such as ticket acquisition, reliable transporting services, hotel accommodation, dining, tour guidelines, pictures taking and many more. Digital health tourism (also termed as e-health tourism) is part of digital tourism strategic platform that used electronic services including electronic government (e-government), electronic commerce (e-commerce), electronic health (e-health), electronic payment (e-payment) and mobile health (m-health) technologies in justifying the effects of COVID-19 disease, focusing on potential benefits of wearable sensors. However, in Nigeria, over 80 million individuals have Internet access that used wearable sensors for digital skills innovation initiatives. Microsoft (International private organization) aimed to support 25 million people worldwide with digital skills modernization and hopes to convey this intervention to five million people in Nigeria, by creating over 27,000 new digital skill jobs in the next three years across six geopolitical zones. Many research methods have been proposed, but most of these concepts lack geometa data profiling process to empower Nigerian youths in digital health skills acquisition [6] under the current COVID-19 economic trial period. Equally, digital health tourism sector is facing a lot of challenges such as unemployment, high cost of human labor, information overload, cybercrimes, data integrity issues, lack of government regulation plans, as well as inadequate of research funding in identifying socio-economic risk factors towards its practical implementation plans. Besides, pre-processing of digital health tourism geometa data profiles using principal component analysis (PCA) can be a great help to uncover standardized behavior intention factors [7], review recent developments in digital health tourism sector, remove noisy geometa data profiles before carrying out hypothesis testing in high dimensional space. Hypotheses testing can provide alternative solutions on how a test of significance can develop measures to rebuild and restore health tourism sector, so that it can become maintainable and stronger in the future. Additionally, encouraging news is on coronavirus vaccination trials for health tourism sector recovery especially in developing nation like Nigeria. Many experts in this field have recommended key policy priorities in digital health tourism initiatives including restoring travelers' confidence, supporting tourism businesses to adopt novel changes, promoting local and international tourism safe travel return, providing clear health information to travelers, limiting uncertainty, as well as building more robust and justifiable digital health tourism. These challenges are open for review for a better future. Therefore, capitalizing on digital health tourism can build a strong economy for policy formulation in developing nations. Similarly, research methodology tools and techniques can also be employed in studying research problem systematically. Along with logical assumptions and justifications to answer the following research questions: (i) What are the primary healthcare workers' behavior intention factors that will affect digital health tourism innovation initiatives in the COVID-19 period? (ii) How can mobile health technologies pre-process behavior intention factors in post COVID-19 economic trials? and (iii) In what way could digital health tourism innovation research hypotheses be formulated to use mobile health technologies? Accordingly, the main objective of this study is to observe medical tourist geometa data profiles, develop a digital health tourism behavior intention model to use mobile health technologies in developing Nations like Nigeria. Besides, COVID-19 socio-economic disruption statements are assumed to be open and can connect geometa data profiles accurately, to enable researchers describe the characteristics of health and healthcare tourists and individuals affected by the COVID-19 interruptions seriously affecting the medical tourism sector with unexpected unemployment and lack of small businesses. This sector is potentially facing weakening cycles. World Travel and Tourism Council (WTTC) evaluated 174 million jobs are at risk. Also, science and technology processes can be applied to discover hidden patterns related to behavior intention factors.

\section{Literature Review}

This section introducing geometa data profiling, behavior intention models, digital health tourism innovation initiatives model in COVID-19 vaccination period towards mobile health technology usage for socio-economic growth. At present, a lot of issues are affecting digital health tourism including lack of 24 hours power supply, unreliable geometa data profiles pre-processing, interoperability, lack of adequate ICT facilities and inadequate of research funding 
to enable researchers inform policymakers about digital skill initiative plans. Digital health tourism is among the major industries worldwide with over 7.6 trillion U.S. dollars investments in accommodation and transport, while it is estimated that the allocations for entertainment amounted to 2.3 trillion U.S. dollars. So, it is significant for health tourism sector to use these novel technologies in tightening domestic and international tourism activities. Digital health tourism innovation is associated to content analysis, contextual observations, and situation similarities, as well as organizational management, enjoyment of local and international travelers using ICT and smart technologies. At present, health tourists are expecting a traveling experience that is unique using Internet services, virtual reality processes and use of health and healthcare big data analytics which have been used as a strategic tool for booking hotels, air tickets and tourist activity recognitions. This opens an opportunity for local and international tourism business operators to use this novel technology for socio-economic growth. Also, local health tourism activities are necessary, as an alternative to escape from expensive urban health tourism to simple rural community health tourism services at a reduced cost. Consequently, it may increase the number of trips, change, or influence the traveling geography and re-routes analog health tourism. Therefore, using the Precaution Adoption Process Model (PAMP) in digital tourism health innovation is an opportunity to pre-process geometa data profiles, use mobile health technologies and ICT facilities for development of standard digital health tourism framework.

\subsection{Geometa Data Profiling to use Mobile Health Technologies}

Primary healthcare workers in developing nations are healthcare professionals such as doctors, clinical officers, nurses, midwives, pharmacists, community health workers, drug shop vendors and other professionals attached to primary healthcare systems who prescribe, dispense medication, conduct medical tests and other related activities. Unfortunately, these health and healthcare experts are unable to provide enough quality medical services and had been subject to criticism on treatments and monitoring of chronic diseases including COVID-19 disease. Therefore, the geometa data profiling aims to improve primary healthcare workers' access to universal healthcare that can meet medical tourists' specific needs and likings in developing nations like Nigeria. Three main categories of geometa data profiling processes available in a digital health recommender system, such as, the structured geometa data profiling that validates factors, examine simple basic statistics like minimum and maximum values, means, medians, modes, as well as standard deviation. Second, the content profiling help in finding areas that contain ambiguous null hypothesis. Finally, the relationship geometa data profiling is to understand the connections among different geometa data sets and the stepwise tests for interaction that can make use of mobile health technologies in various domains. However, mobile health is a sub-module of electronic health services, largely explored to mitigate the effects of the disease especially now during COVID-19 economy [8], health and healthcare professionals are focusing on potential benefits of wearable sensors in providing pervasive and patient-centered care that may support them in delivering short text messaging services (SMS), emails, and applying social media services in an active medical research area commonly used in both developed nations. Additionally, mobile health facility functionalities include general packet radio service (GPRS), third generation (3G), fourth generation (4G) communication services, the global positioning system (GPS) and Bluetooth technology and other health sensing devices are used to provide public health practice support and reduce healthcare contextual and technical health and healthcare barriers. Figure 1. shows digital health tourism innovation geometa data profiling using mobile health technologies describing the user, location, noisy environment, domain activity, user role, schedule, contacts, event, service access and service profiles in rural and urban communities.

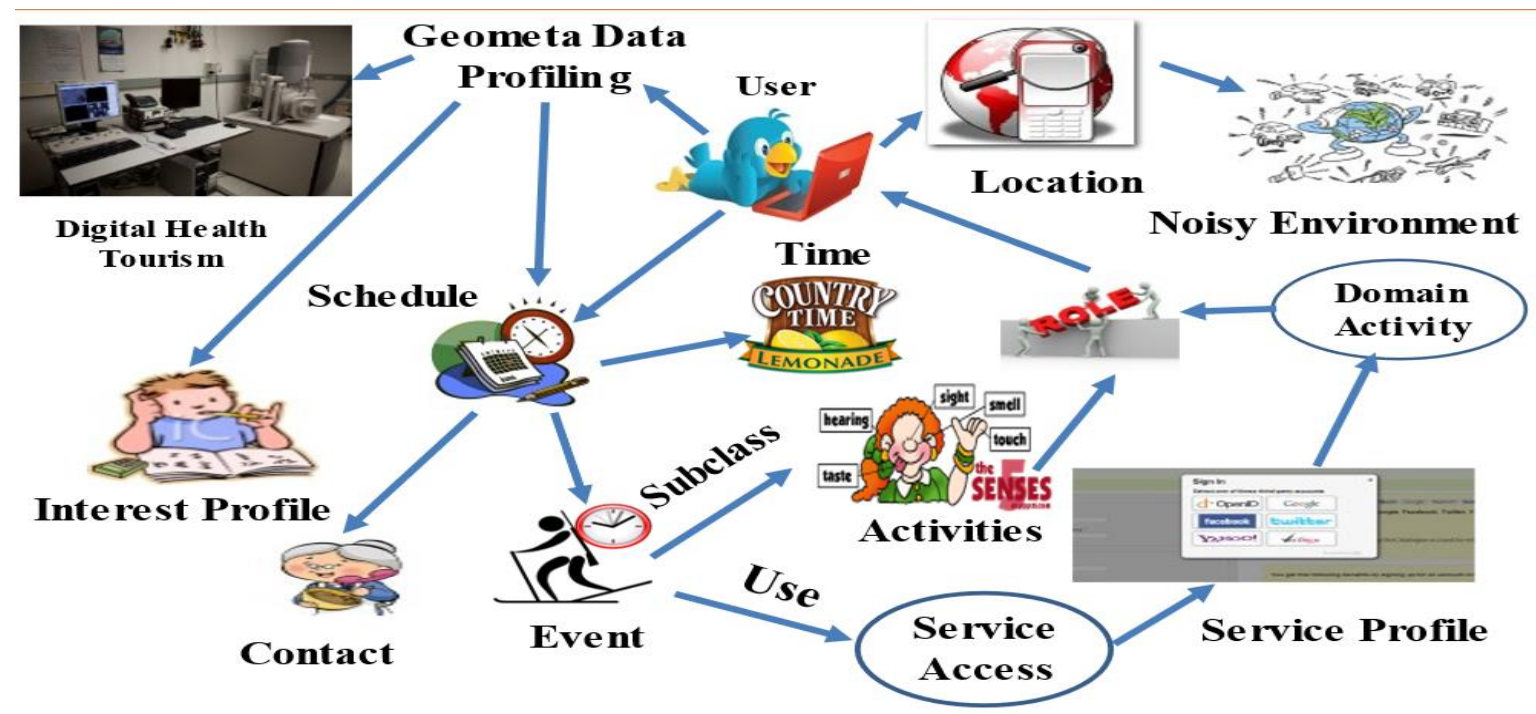

Fig. 1 - The proposed digital health tourism geometa data profiling 


\subsection{Theoretical Background of Behaviour Intention Models}

Behaviour intention model perceptions has shown that many behaviour intention theories and empirical studies such as theory of planned behaviour (Ajzen, 1991, 2006) and theory of reasoned action (Fishbein \& Ajzen, 1975) used digital health tourism innovation practices to pre-processed bioinformatic behavior intention factors, mobile forensic services to regulate background behaviors for information sharing, recognise the value of health information technology services, and distribute significant health information that can impact behaviors across many disciplines, using innovative approaches against diseases. Also, measure significance relationship among government electronic services taking up. In addition, these processes are to avoid or control bioattack, cybersecurity issues, spread of viruses, internal and external movement, and improve provision of social amenities such as stable power supply, good transporting system and portable water supply. Similarly, ground up exploration approach has been piloted in Nigeria using qualitative research approach only to understand use of mobile technologies (mobile phone) for assessing primary healthcare professionals' attitudes. Similarly, behavior intention theories such as theory of planned behaviour (TPB) and social cognitive theory (SCT) are to describe behaviour intention factors for information security from working environments, observe pre-processed health and healthcare data gathering for health professional rehabilitation awareness. Academic research professionals and primary healthcare workers can adopt, adapt behaviour intention factors towards digital health tourism innovation theoretical and empirical exploration to use in public and private health and healthcare sectors.

\subsection{Expert Validation of Behaviour Intention Theoretical Factors and Factor Items}

Eight academic researchers who were conversant with digital health technology validated 28 behavior intention factors with average factor loading values of 50\% to 75\%. Pilot survey conducted among 700 respondents from March 18,2020 , to September 10, 2021, among them are undergraduate students that may use this technology for research purposes. Pre-processed geometa data have shown percentage frequency counts of internet access and other online services $8 \%$ to $95 \%$, adapted training factors $49 \%$ to $92 \%$ and factor items $34 \%$ to $78.3 \%$ for hypothesis generation towards development of digital health tourism framework in finding explanation to COVID-19 economic challenges. Except behavior intention factors and factor items insights are known and mapped, mobile health technology design process may result in poor conclusions. Thus, patients recovered from COVID-19 infection can still be infected again.

\subsection{Digital Health Tourism Conceptual Framework Generation}

Digital health tourism innovation PHWs mobile health conceptual framework is based on the proposed PAMP, made up of seven levels for processing geometa data profiles to use mobile health technologies and observe behavior intention factors and factor items. However, not all behavior intention factors, and factor items fit the seven levels of the PAMP model. Some factors used compound strategies. PAPM may be good enough to identify medical tourist problems and achieve research objectives using 7 levels as follows.

- Level One: Lack of awareness, and inadequate of research funding to support the use of digital health tourism mobile health innovation initiative plans.

- Level Two: Academic research experts validated behavior intention theoretical factors with average loading factor values.

- Level Three: PHWs at this level feel the need to join digital health tourism activities to support information sharing, and cybercrime monitoring to avoid victimology.

- Level Four: Initial use of mobile health technologies, perceived benefits, information sharing, use of ICT facilities and AI attachment are to support digital health tourism innovation processes during post COVID-19 period.

- Level Five: PHWs and other professionals perceived benefits of digital health tourism to use mobile health technologies during COVID-19 vaccination trials.

- Level Six: PHWs and other professionals will actively be participating in health and healthcare activities using the proposed Digital health tourism innovation initiatives in Nigerian local governments. 
- Level Seven: PHWs and other professionals may continue to use digital health tourism using mobile health technologies in finding an alternative solution to post COVID-19 and other diseases condition especially at the primary healthcare system in Nigeria's local government.

\subsubsection{Digital Health Tourism Hypotheses Generation}

Correspondingly, the proposed digital health tourism behavior intention framework has six hypotheses from the 28 validated behavior intention factors in form of scholarly assumptions. Therefore, standard adapted model constructs are to be connected at different levels to give independent variables connection measures and dependent variables outcomes. Initial understanding of $\mathrm{m}$-health, perceived benefits, perceived threats, attitude towards behavior, information sharing, ICT infrastructure, attachment and behavior intention are the proposed digital health tourism behavior intention model constructs to use $\mathrm{m}$-health technologies. Understanding of behavior intention factors from geometa data profiles is very significant to use $\mathrm{m}$-health technologies. Figure 3 illustrates the digital health tourism behavior intention framework to use mobile health technologies in Nigerian.

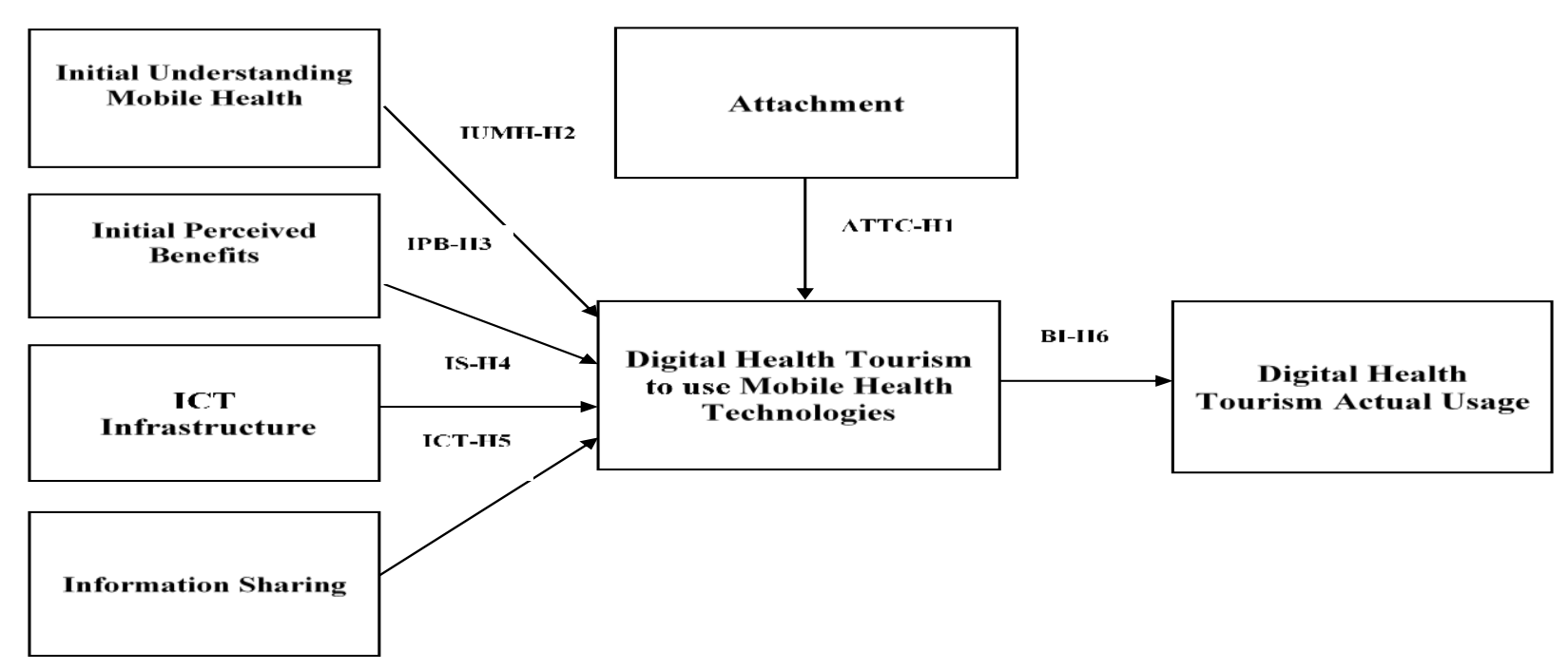

Fig. 3 - The proposed digital health tourism framework in Nigerian local governments

In addition, building from cited literature attachment, initial understanding of mobile health technologies, initial perceived benefits, information sharing, and ICT Infrastructure are hypothesized as follow:

\subsubsection{Attachment}

Attachment is a specific interest an individual has with co-workers, supervisors, jobs, and organizations. In this study, it is posited to has a positive effect on behavior. Therefore, attachment is hypothesized as: ATTC-H1. The attachment will significantly affect digital tourism PHWs BI mobile health services towards compliance with necessary guidelines of information sharing and information security.

\subsubsection{First Impression Factors}

Initial understanding of mobile health, perceived benefits are first impression factors of health and healthcare professionals towards mobile health technologies these factors are hypothesized as IUMH-H2: Initial understanding of mobile health will significantly affect digital health tourism to use mobile health technologies. IPB-H3: Initial perceived benefits will significantly affect digital health tourism model to use mobile health technologies.

\subsubsection{Information Sharing}

Information sharing systems to medical tourists, healthcare professionals and the public can allow free flow of health information. Hence, information sharing is hypothesized as:IS-H4: Information sharing will significantly affect digital tourism PHWs BI model to use of m-health technologies. 


\subsubsection{ICT Infrastructure}

In the Nigerian context, digital health tourism needs ICT infrastructure for sustainable procedure of healthcare information systems to premeditated health tourist activities. Therefore, ICT infrastructure is hypothesized as: ICT-H5: ICT infrastructure will significantly affect digital tourism PHW' behavioral intention to use mobile health technologies.

\subsubsection{Behavior Intention}

Behavior Intention is health tourist conscious plans to perform or not to perform some specified future behaviors using mobile health towards its actual usage. Thus, primary healthcare workers behavior intention model is hypothesized as: BI-H6: PHWs' behavior intention will significantly affect the actual use of mobile health technologies.

\subsection{Coronavirus (COVID-19) Vaccination Trials}

Coronaviruses (COVID-19) disease look a lot like sun's coronavirus, infecting and killing millions of people worldwide more than that of severe acute respiratory syndrome (SARS) pandemic. At present, ICT academic researchers, health and healthcare professionals don't know where COVID-19 matters going to end particularly due to serious unseen health and healthcare conditions such as diabetes, asthma, cancer, HIV/AIDS, and heart disease, and stroke cases. Similarly, "anxiety was connected with COVID-19 pandemic vaccines through ID 2020, Internet of things (IOT) and next generation technologies such as 3G, 4G,5G and beyond, threatening the peace of local and international groups, taking complete control of domain socioeconomic matters, spiritual or moral activities. "COVID19 pandemic will be crushed not only in United State of America but worldwide." Nevertheless, no COVID-19 vaccine disturbs fertility. Vaccination trials require precise digital health tourism management accompanied with thorough safety assessment and immune retorts. However, tackling COVID-19 vaccine test trials can significantly support digital health tourism policies. These, coronavirus vaccination trials include COVID-19 vaccine progress, Its delivery, and positioning. Decision makers can expect and identify these encounters and plan alternative solution to augment to the upright weapons against the spread of COVID-19 diseases.

\subsection{Research Methodology}

Research 'methodology, refers to 'method'+'ology.' Ology means a self-control branch of knowledge. It is the study of research methods. We simply mean the research techniques or tools to be used for conducting digital technology innovation research irrespective of whether the study belongs to physical or social sciences or any other disciplines. In this research paper, geometa data profiling, behaviour-intention theoretical factors were reviewed, geometa data profiles were observed and Expert validated behavior intentions factors and factor items. Qualitative and quantitative methods (mixed method) were employed to pre-process experts confirmed behavior-intention factors. A pilot survey was conducted using the Proposed Precaution Adoption Model Process (PAMP) questionnaire in finding explanation to primary-healthcare workers theoretical and empirical practice plans. As a result, these factors may be used to develop research methodologies taxonomy of coronavirus (COVID-19) interruption in Nigeria. At present, there is a limited number of research to describe the use of mobile health technologies, distribute health and healthcare information for information security, cybercrime monitoring, self-diet tracking, and physical health fitness through theoretical and empirical practices.

Nevertheless, academic ICT researchers, health and healthcare professionals, and other experts in their disciplines employ scientific tools and techniques that may differ widely in nature and complexity. Generally, digital health tourism researchers adopted various research methodologies in studying research problems systematically, along with logic, assumptions, justification, and rationale behind them, seeks to answer research questions to achieve research objectives. Thus, when we speak of research methodology, we are not only talking of research methods, but also logic and justification in the context of our research undertaking in acquiring knowledge to perform tests, experiments, surveys, and critical analysis. Ensures, deployment of correct procedures to solve research problems and paves way to choose suitable research methods in the beginning of any research of information system, and draw conclusions on how to use mobile health technologies that can identify research methods, answer research questions and to achieve research objectives, consider study ethics and limitations towards successiful implementation. Also be viewed as the discipline of validating and accepting applicable research methods to manage any form of research investigation.

\section{Discussion}

Digital health tourism geometa data profiling to use mobile health technologies is for the implementation of the proposed digitized recommender system that can provide research focus towards positive digital health tourism development plans in identifying many factors in different domains. Although, this study is to support travel agents, analyze health and healthcare professionals and general populace by observing their favorites, financial plan, 
whereabouts, dining habits, and understand the real situation based on the present and past experiences in selecting mobile health technologies for electronic health record integration, and digital skills attainment among youths. This study used geometa data profiling pilot survey analysis using PAMP questionnaire for self-alleged health, lifespan fulfillment and correlated behavior intention factors collection among healthcare experts and wide-ranging population for development of score values adjustments and medical tourism theory integration that can protect medical tourist with immunity passport for COVID-19, since those tested have shown to be immune can be more willing to engage with health and healthcare intervention plans, practiced better health and healthcare in the longer term [10]. The pilot survey analysis used PAMP questionnaire, a 5-point Likert-type towards using mobile health technologies. Therefore, independent variables and dependent variables are to be considered for the measurement of behavior intention factor constructs and hypotheses testing. Besides, a minimum sample size of 50,100 are considered when a model contained five or fewer expert' confirmed behavior intention factor constructs. Also, 150 sample sizes are observed when a conceptual model is containing seven or fewer expert confirmed factors. Equally, 500 samples can be used when a model contains many experts' validated factors [11]. Also, a sample size of 150 may be used in factor analysis using principal components analysis to evaluate mobile health parameters that is usually done in the design stage to set the record straight [12].

\section{Conclusion}

Practical applications of geometa data profiling and overall model performance are significantly required to fill the theoretical and empirical research gaps, handle mislaid data using statistical models, approximate distributed errors and overcome other unknown difficulties. In total five behavior intention theoretical factors were identified and validated towards hypotheses testing and statistical analysis. These factors include initial understanding of $\mathrm{m}$-health $(0.75)$, initial perceived benefits (0.63), information sharing (0.63), ICT infrastructure (0.63), and attachment (0.63). The proposed digital health tourism model to use mobile health can make a positive significant influence on socio-economic development initiatives and can be utilized towards managerial participation, ecology, country dimensions assessment and digital skills innovation development program plans. Nevertheless, we are to judge factor item loading average values for convergent validity, composite reliability, Cronbach's Alpha, and predictive validity. Cut off loading average values can be more than 0.70 , are more important. However, if loading factor item average value is higher than 0.50 , then between 0.50 and 0.70 are tolerable [11].

\subsection{Digital Health Tourism Research and Practical Implications}

The participant population of the study was limited to only 700 Nigerian citizens across six political zones among are undergraduates in higher tertiary institutions, that may use this digital technology innovation initiative for research purposes. Therefore, the results cannot be generalised to all people in Nigeria. Moreover, the study contributes to existing knowledge on adoption and adaption of digital technology particularly digital health tourism geometa data profiling framework to use mobile health technologies and enable authorization of digital technology innovativeness among general populace, behavior intention model constructs should be taken into account, and Nigeria citizens should be encouraged to participate for full implementation to save cost, time for socio-economic development.

\section{Acknowledgments}

This work was initiated by the corresponding author; Abdullahi Hassan Birnin-kudu, a researcher from Faculty of Computing and Information Technology, Federal University Dutse, Jigawa State, Nigeria, supported by Professor Dr. Wan Rozaini Sheik Osman and Dr. Hapini Awang from School of Computing, Universiti Utara Malaysia, 06010 Sintok Kedah. All rights reserved.

\section{References}

[1] Ferri-Garcia, R., Rueda, M-M., and Cabrera-Leon, A. (2021). Self-Perceived Health, Life Satisfaction and Related Factors among Healthcare Professionals and the General Population: Analysis of an Online Survey, with Propensity Score Adjustment. Mathematics 9:7, pages 791

[2] Nguyen, T-M-T., Dang, H-S., Wan, C-N., Day, J-D and Dang T-M-H. (2020). Grey System Theory in the Study of Medical Tourism Industry and Its Economic Impact. International of Medical Tourism 2020.17,961; doi:10.3390/ijerph17030961

[3] Dalen, J. E., and Alpert, J. S. (2019). Medical Tourists: Incoming and Outgoing. The American Journal of Medicine Vol. 132 (1) pp 9-10 doi.org/10.1016/j.amjmed.2018.06.022.

[4] Iyamu, T. (2020). A framework for selecting analytics tools to improve healthcare big data usefulness in developing countries. South African Journal of Information Management (SAJIM). Vol.22 n.1 2020 ISSN 1560683X, 2078-1865.: dx.doi.org/10.4102/sajim. v22i1.1117.

[5] Gaines, J., and Lee, C. V. (2016). Medical Tourism. In Travel Medicine. Elsevier: Oxford; 2016 pp 371-375 
[6] Akwaja, C. (2021). NCC Lauds 9mobile for Promoting Digital Skills among Nigerian Youths. 2020 Leadership Conference \& Awards. Available at https://leadership.ng/ncc-lauds-9mobile-for-promoting-digital-skilss-amongnigerian-youths/amp/

[7] Forkman J Josse J and Piepho H P 2019 Hypothesis tests for principal component analysis when variables are standardized J. Agric. Biol. Environ. Stat. 24 289-308.

[8] Adans-Dester, C., Bamberg, S., et al. (2020). Can mHealth Technology Help Mitigate the Effects of the COVID19 Pandemic? IEEE Open Journal of Engineering in Medicine and Biology, 2020; 1 DOI: 10.1109/OJEMB.2020.3015141

[9] Shaw, R., Stroo, M., Fiander, C., and McMillan K. (2020). Selecting Mobile Health Technologies for Electronic Health Record Integration: Case Study. Journal of Medical Internet Research 2020; 22(10): e23314 doi:10.2196/23314 PMID: 33112248 PMCID: 7657715

[10] Brown, R. C. H., Savulescu, J., Williams, B., and Wilkinson, D. (2020). Passport to freedom? Immunity passports for COVID-19. Journal of Medical Ethics Vol. 46 (10) pp 652-659.: dx.doi.org/10.1136/medethics-2020-106814

[11] Hair, J. F., Howard, M. C., \& Nitzl, C. (2020). Assessing measurement model quality in PLS-SEM using confirmatory composite analysis. Journal Business Research 109 (1): 101-110.: doi.org/10.1016/j.jburres.2019.11.069

[12] Schuberth, F. (2021). Confirmatory composite analysis using partial least squares: setting the record straight. Rev Management Science 15, 1311-1345 (2021).: doi.org/10.1007/s11846-020-00405-0 